\title{
Intracranial Hypertension Syndrome Revealing Cerebral Thrombophlebitis in a Patient treated for Breast Cancer by Tamoxifen after Chemotherapy
}

\author{
Karima Oualla ${ }^{1 *}$, Samia $\quad 1$, Sihame Tizniti ${ }^{2}$ and Omar EI Mesbahi ${ }^{1}$
}

${ }^{1}$ Department of medical oncology, Hassan Il university hospital Fez, Morocco

${ }^{2}$ Department of radiology; Hassan II university hospital Fez, Morocco

\begin{abstract}
Cancer is a major risk factor for thromboembolic disease. This risk is increased by the therapies applied including hormone therapy. We report a case of a 54 years old patient, postmenopausal, treated for breast cancer who presented an intracranial hypertension syndrome 15 months after starting Tamoxifen. A brain scan with injection of contrast was performed and revealed a left lateral sinus thrombophlebitis extended to the ipsilateral jugular vein. Curative anticoagulation was started the same day of diagnosis with low molecular weight heparin. Symptoms were improved 5 days after starting anticoagulation.
\end{abstract}

Keywords: Breast Cancer; Tamoxifen; Cerebral Thrombophlebitis

Abbreviations: DVT: Deep Venous Thrombosis; PE: Pulmonary Embolism; CVT: Cerebral Venous Thrombosis

\section{Introduction}

Cancer is a major risk factor for thromboembolic disease, including deep vein thrombosis and pulmonary embolism. Tamoxifen therapy is reported to increase the risk of deep venous thrombosis which can involve all areas including the venous cerebral network. This localization remains very rare $[1,5,7]$.

\section{Case presentation}

We report the case of a 54 years old patient, postmenopausal, treated for breast cancer in the department of medical oncology at Hassan II University hospital. Her past medical history was significant with breast cancer in her maternal aunt. The patient received radical mastectomy with lymphadenectomy. Histology revealed infiltrative ductal carcinoma, with metastatic nodes. Immunohistochemical evaluation was positive for hormone receptors (estrogen receptor $12 \%$, progesterone receptor $60 \%$ ) and negative for Her $2 /$ neu. Bone metastases were discovered after surgery.

The patient received 6 cycles of chemotherapy by doxorubicin and cyclophosphamide with good clinical and biological tolerance followed by treatment with tamoxifen. 15 months later, the patient presented headache with vomiting and visual mists. A brain scan revealed, after injection of contrast, thrombophlebitis of the left lateral sinus extended to the jugular vein (Figure 1).

After the diagnosis of cerebral thrombophlebitis, new bone lesions were discovered and controlateral breast lesion was found in mammography and confirmed by biopsy. Anticoagulation was started the day of diagnosis with low molecular weight heparin.

\section{Discussion}

Cerebral venous thromboses are uncommon in cancer [1]. This localization is rare and must be raised before appeal neurological signs simulating the occurrence of cerebral metastasis.

Survival of patients with cancer experiencing venous thrombosis seems to be worse. It is the second cause of death among cancer patients [2].

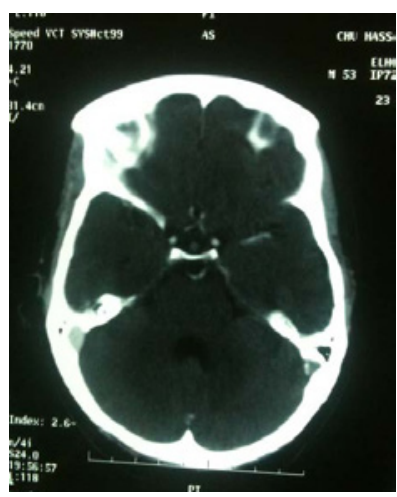

Figure 1: Brain scan with injection of contrast: thrombophlebitis of the left lateral sinus extended to the jugular vein.

The association of cancer and venous thromboembolic disease has been described for the first time in the late nineteenth century. It was called Trousseau's syndrome [3].

Physiopathology of this association, explained by acquired hypercoagulability due to a multifactorial process involving the activation pathways of coagulation and fibrinolytic system, the procoagulant activity of tumor cells, and interaction of tumor cells with blood cells and endothelium, and decreased natural anticoagulant activity.

Moreover, there is a genetic link between cancer and thrombosis. The activation of blood coagulation results in a selective advantage for

*Corresponding author: Karima Oualla, Department of medical oncology, Hassan II university hospital Fez, Morocco, Tel: 002126766102 67; E-mail: karimarauf@ hotmail.com

Received June 27, 2011; Accepted September 05, 2011; Published September 13, 2011

Citation: Oualla K, Arifi S, Tizniti S, El Mesbahi O (2011) Intracranial Hypertension Syndrome Revealing Cerebral Thrombophlebitis in a Patient treated for Breast Cancer by Tamoxifen after Chemotherapy. J Cancer Sci Ther 3: 195-196. doi:10.4172/1948-5956.1000087

Copyright: (C) 2011 Oualla K, et al. This is an open-access article distributed under the terms of the Creative Commons Attribution License, which permits unrestricted use, distribution, and reproduction in any medium, provided the original author and source are credited. 
Citation: Oualla K, Arifi S, Tizniti S, El Mesbahi O (2011) Intracranial Hypertension Syndrome Revealing Cerebral Thrombophlebitis in a Patient treated for Breast Cancer by Tamoxifen after Chemotherapy. J Cancer Sci Ther 3: 195-196. doi:10.4172/1948-5956.1000087

cancer cells, as fibrin provides a scaffold for anchorage and invasion, and coagulation proteins induce receptor-mediated intracellular signals promoting invasive growth [4].

In addition to cancer treatments initiated increase the risk of thromboembolism: surgery, chemotherapy, radiation therapy and hormone therapy [5,6]. Tamoxifen is one of hormone therapies indicated for the treatment of hormone sensitive breast cancer. It increases venous thromboembolic risk among postmenopausal women. It has been associated with an increased risk of thromboembolic events, including deep vein thrombosis, pulmonary embolism and cerebral vascular accident. This frequency is likely to increase when tamoxifen is administered in combination with chemotherapy.

The Danish population-based cohort study has showed that the first 2 years after the initiation of tamoxifen therapy may be the most crucial time for monitoring DVT/PE risk, particularly in older women [5].

In the Eastern Cooperative Oncology Group (ECOG), studies of adjuvant therapy for breast cancer were reviewed for the occurrence of vascular complications in order to compare the frequency of vascular complications among patients who received adjuvant therapy for breast cancer with patients on observation, and to estimate the contribution of chemotherapy and hormonal therapy to the occurrence of venous and arterial thrombi. The results showed that patients who received adjuvant therapy for breast cancer had a $5.4 \%$ frequency of thromboembolic complications, significantly more than those who were observed [6]. The combination of chemotherapy and tamoxifen was associated with more venous thrombosis complications than chemotherapy alone in premenopausal patients.

Paraneoplasic cerebral venous thrombosis (CVT) was reported in the literature about twenty cases in hematological adults, particularly in treatment of acute lymphoblastic leukemia (two thirds) and lymphomas [7-9]. It appears mainly during the introduction of chemotherapy.

However, in solid tumor adults CVT is relatively rare. Only 12 cases of paraneoplastic CVT were reported in the literature [3,8-12]. The frequency of the events mentioned above was evaluated in the context of a Long-term clinical trial on prevention of breast cancer, known as the National Surgical Adjuvant Breast and Bowel Project (NSABP P-1). Under this test, patients exposed to high risk were randomized into 2 groups and have received tamoxifen or placebo. The risk of cerebral vascular accident, pulmonary embolism and venous thrombosis was respectively 1.6, 3.0 and 1.6.

The established treatment for cancer-associated thrombosis is anticoagulant therapy. Low molecular weight heparin (LMWH) is the preferred agent because of its efficacy, safety, and convenience. The use of daily subcutaneous LMWH for at least three to six months is recommended as first line therapy [13].

However anticoagulant prophylaxis is not recommended in all ambulatory cancer patients because of few trials in this way. Research is needed to better define a population at high enough risk to warrant pharmacologic prophylaxis and to develop new antithrombotic agents for this indication [14].

\section{Conclusion}

Cerebral localization of venous thrombosis is uncommon in cancer. It must be raised before neurological signs simulating the occurrence of cerebral metastases.

\section{Funding}

This work received no specific grant from any funding structure.

\section{Acknowledgment}

I acknowledge anyone who contributed towards this study.

\section{References}

1. Hickey WF, Garnick MB, Henderson IC, Dawson DM (1982) Primary cerebral venous thrombosis in patients with cancer- a rarely diagnosed paraneoplastic syndrome. Report of three cases and review of the literature, Am J Med 73 740-750.

2. Sutherland DE, Weitz IC, Liebman HA (2003) Thromboembolic complications of cancer: epidemiology, pathogenesis, diagnosis, and treatment. Am J Hematol 72: 43-52.

3. Trousseau A (1865) Phlegmasia alba dolens, Clinique médicale de l'HôtelDieu de Paris 3: 654

4. Boccaccio C, Comoglio PM (2009) Genetic link between cancer and thrombosis Clin Oncol 4827-4833.

5. Hernandez RK, Sørensen HT, Pedersen L, Jacobsen J, Lash TL (2009) Tamoxifen treatment and risk of deep venous thrombosis and pulmonary embolism: a Danish population-based cohort study. Cancer 115: 4442-4449.

6. Saphner T, Tormey DC, Gray R (1991) Venous and arterial thrombosis in patients who received adjuvant therapy for breast cancer. J Clin Oncol 9: 286294.

7. Meininger V, James JM, Rio B, Zittoun R (1991) Dural venous sinus occlusions in hemopathies. Rev Neurol 141: 228-233.

8. Sigsbee B, Deck MD, Posner JB (1979) Non-metastatic superior sagittal sinus thrombosis complicating systemic cancer. Neurology 29: 139-146.

9. Raizer JJ, DeAngelis LM (2000) Cerebral sinus thrombosis diagnosed by MRI and MR venography in cancer patients. Neurology 54: 1222-1226.

10. EEllie E, Becouarn Y, Brunet R, Gonday G, Julien J (1993) Thrombose veineuse cérébrale paranéoplasique, Rev. Neurol 149:297-299.

11. Graus F, Rogers LR, Posner JB (1985) Cerebral complications in patients with cancer. Medicine 64: 16-35.

12. Preter M, Tzourio C, Ameri A, Bousser MG (1996) Long-term prognosis in cerebral venous thrombosis. Follow-up of 77 patients. Stroke 27: 243-246.

13. Lee AY (2009) Anticoagulation in the Treatment of Established Venous Thromboembolism in Patients With Cancer. J Clin Oncol 4895-4901.

14. Rana P, Levine MN (2009) Prevention of Thrombosis in Ambulatory Patients With Cancer. J Clin Oncol 4885-4888. 